\title{
SOME FURTHER PROPERTIES OF A q-ANALOGUE OF MACROBERT'S E-FUNCTION
}

\author{
by R. P. AGARWAL \\ (Received 7 November, 1961)
} the form

1. Introduction. Recently, I gave an analogue [1] of the MacRobert's $E$-function [4] in

$$
E_{q}(\alpha, \beta:: z)=\sum_{\alpha, \beta} \frac{G(\alpha) G(\beta-\alpha)}{G(1)} \prod_{n=0}^{\infty} \frac{\left(1+z^{-1} q^{\alpha+n}\right)\left(1+z q^{1-\alpha+n}\right)}{\left(1+z^{-1} q^{n}\right)\left(1+z q^{1+n}\right)}, \Phi_{1}\left(\alpha ; \alpha-\beta+1 ; z q^{2-\beta}\right),
$$

where the symbol $\sum_{a, \beta}$ denotes that a similar expression with $\alpha$ and $\beta$ interchanged is to be added to the expression following it. It has since then been generalized by N. Agarwal [2], who defined and studied the $q$-analogue of the generalized $E$-function. In this paper I give some further properties of the $E_{q}$-function.

2. Notation and definitions. The following notation is used throughout the paper. Let $|q|<1$,

$$
\begin{gathered}
\left(q^{a}\right)_{n} \equiv(a)_{n}=\left(1-q^{a}\right)\left(1-q^{a+1}\right) \ldots\left(1-q^{a+n-1}\right), \\
\left(q^{a}\right)_{0}=1, \quad\left(q^{a}\right)_{-n}=(-)^{n} q^{i n(n+1)} q^{-n a} /\left(q^{1-a}\right)_{n} ;
\end{gathered}
$$

then we define the generalized basic hypergeometric function as

$$
{ }_{r+1} \Phi_{r}\left[\begin{array}{l}
a_{1}, a_{2}, \ldots, a_{r+1} ; z \\
b_{1}, b_{2}, \ldots, b_{r}
\end{array}\right]=\sum_{n=0}^{\infty} \frac{\left(a_{1}\right)_{n} \ldots\left(a_{r+1}\right)_{n}}{(q)_{n}\left(b_{1}\right)_{n} \ldots\left(b_{r}\right)_{n}} z^{n}
$$

and the "confluent" basic hypergeometric function as

Also

$$
{ }_{1} \Phi_{1}(a ; b ; z)=\sum_{n=0}^{\infty} \frac{(a)_{n}}{(q)_{n}(b)_{n}} q^{\frac{1 n(n-1)}{z^{n}}}
$$

and

$$
\begin{aligned}
E_{q}(x) & =\prod_{n=0}^{\infty}\left(1-x q^{n}\right)=\sum_{n=0}^{\infty} \frac{(-)^{n} q^{\frac{j n(n-1)}{}}}{(q)_{n}} x^{n}, \\
e_{q}(x) & =1 / \prod_{n=0}^{\infty}\left(1-x q^{n}\right), \\
(x+y)_{\alpha} & =x^{\alpha}\left(1+\frac{y}{x}\right)_{\alpha}=x^{\alpha} \prod_{n=0}^{\infty}\left(\frac{1+y x^{-1} q^{n}}{1+y x^{-1} q^{\alpha+n}}\right), \\
G(\alpha) & =\left\{\prod_{n=0}^{\infty}\left(1-q^{\alpha+n}\right)\right\}^{-1} .
\end{aligned}
$$

The basic-differential operator $q^{\theta}$ is defined by $q^{\theta} z(x)=q^{(x d / d x)} z(x)=z(q x)$, where $q^{(x d / d x)}$ means $\exp \left(x \frac{d}{d x} \log q\right)$. 
Further, following Hahn [3], the basic integral of a function, under suitable conditions, is defined as

and thus

$$
\begin{aligned}
& {\underset{\mathrm{S}}{0}}_{0}^{x} f(y) d(q y)=x(1-q) \sum_{i=0}^{\infty} q^{i} f\left(q^{i} x\right), \\
& {\underset{x}{x}}_{x}^{\infty} f(y) d(q y)=x(1-q) \sum_{j=1}^{\infty} q^{-j} f\left(q^{-j} x\right),
\end{aligned}
$$

$$
{\underset{S}{0}}_{0}^{\infty} f(y) d(q y)=(1-q) \sum_{j=-\infty}^{\infty} q^{j} f\left(q^{j}\right)
$$

All products occurring are infinite products so that, for example, $\prod_{n=0}^{\infty}\left(1-x q^{n}\right)$ is written as $\prod\left(1-x q^{n}\right)$.

3. A difference-equation satisfied by $E_{q}(\alpha, \beta:: z)$. Let

$$
S=\prod \frac{\left(1+z^{-1} q^{a+n}\right)\left(1+z q^{1-\alpha+n}\right)}{\left(1+z^{-1} q^{n}\right)\left(1+z q^{1+n}\right)} .
$$

Then it is easy to see that

$$
\left(q^{\theta}-q^{\alpha}\right) S=0
$$

Also,

$$
\omega={ }_{1} \Phi_{1}\left(\alpha ; \alpha-\beta+1 ; z q^{2-\beta}\right)
$$

satisfies the $q$-difference equation

$$
z q^{2-\beta}\left(1-q^{\theta+\alpha}\right) q^{\theta} \omega=\left(1-q^{\theta}\right)\left(1-q^{\theta+\alpha-\beta}\right) \omega .
$$

Putting $v=\omega S$, we have, after slight calculations,

$$
z q^{2-\alpha-\beta}\left(1-q^{\theta}\right) q^{\theta} v=\left(1-q^{\theta-\alpha}\right)\left(1-q^{\theta-\beta}\right) v \text {. }
$$

Hence

$$
v=\prod \frac{\left(1+z^{-1} q^{\alpha+n}\right)\left(1+z q^{1-\alpha+n}\right)}{\left(1+z^{-1} q^{n}\right)\left(1+z q^{1+n}\right)}{ }_{1} \Phi_{1}\left(\alpha ; \alpha-\beta+1, z q^{2-\beta}\right)
$$

satisfies the difference equation (3). Since (3) is symmetrical in $\alpha$ and $\beta$,

$$
\prod \frac{\left(1+z^{-1} q^{\beta+n}\right)\left(1+z q^{1-\beta+n}\right)}{\left(1+z^{-1} q^{n}\right)\left(1+z q^{1+n}\right)}{ }_{1} \Phi_{1}\left(\beta ; \beta-\alpha+1 ; z q^{2-\alpha}\right)
$$

also satisfies (3). Hence (3) gives the $q$-difference equation satisfied by $E_{q}(\alpha, \beta:: z)$.

4. A contour integral representation for $E_{q}(\alpha, \beta:: z)$. Consider the contour integral

where $q=e^{-t}, t>0$.

$$
\frac{t}{2 \pi i} \int_{-i \pi / t}^{i \pi / t} \prod\left[\frac{\left(1+q^{\alpha-z+s+n}\right)\left(1+q^{1+z-\alpha-s+n}\right)}{\left(1-q^{\alpha+s+n}\right)\left(1-q^{\beta-\alpha-s+n}\right)\left(1-q^{-s+n}\right)}\right] d s,
$$


Evaluating the integral by the calculus of residues, $\uparrow$ we find that (4) is equal to

$$
\prod\left[\frac{\left(1+q^{n-z}\right)\left(1+q^{1+n+z}\right)}{\left(1-q^{n+1}\right)^{2}}\right] E_{q}\left(q^{\alpha}, q^{\beta}:: q^{z}\right) \text {. }
$$

Writing $z, \alpha, \beta$ for $q^{z}, q^{\alpha}, q^{\beta}$, respectively, we get the required result.

5. Two definite integral representations for $E_{q}(\alpha, \beta:: z)$. Consider the known integral $[3$, p. 290]

$$
\begin{gathered}
\stackrel{S}{0}_{0}^{\infty} e_{q}(-s x) x^{\beta-1}{ }_{1} \Phi_{1}\left(q^{\alpha} ; q^{\gamma} ;-t x\right) d(q x) \\
=(1-q)_{\beta-1} \prod \frac{\left(1+q^{\beta+n} s\right)\left(1+s^{-1} q^{1-\beta+n}\right)}{\left(1+s q^{n}\right)\left(1+s^{-1} q^{1+n}\right)}{ }_{2} \Phi_{1}\left(q^{\alpha}, q^{\beta} ; q^{\gamma} ;-t q^{\beta} / s\right) .
\end{gathered}
$$

Letting $\gamma \rightarrow \infty, s \rightarrow 1$ and $t=1 /\left(z q^{\beta}\right)$ in (5), we have

$$
\begin{gathered}
\quad \operatorname{S}_{0}^{\infty} e_{q}(-x) x^{\beta-1} \Phi_{1}\left(q^{\alpha} ; 0 ;-x q^{-\beta} / z\right) d(q x) \\
=(1-q)_{\beta-1} \prod \frac{\left(1+q^{\beta+n}\right)\left(1+q^{1-\beta+n}\right)}{\left(1+q^{n}\right)\left(1+q^{1+n}\right)}{ }_{2} \Phi_{0}\left(q^{\alpha}, q^{\beta} ;-1 / z\right) .
\end{gathered}
$$

Now, since we know that, $\ddagger$ when $|z|>1$,

$$
{ }_{2} \Phi_{0}\left(q^{\alpha}, q^{\beta} ;-1 / z\right)=\sum_{\alpha, \beta} \frac{G(\beta-\alpha)}{G(\beta)} \prod \frac{\left(1+z^{-1} q^{\alpha+n}\right)\left(1+z q^{1-\alpha+n}\right)}{\left(1+z q^{1+n}\right)\left(1+z^{-1} q^{n}\right)}{ }_{1} \Phi_{1}\left(q^{\alpha} ; q^{1+\alpha-\beta} ; z q^{2-\beta}\right)
$$

we have, for $R(\beta)>0$ and $|z|>1$,

$$
\prod \frac{\left(1+q^{\beta+n}\right)\left(1+q^{1-\beta+n}\right)\left(1-q^{\alpha+n}\right)}{\left(1+q^{n}\right)\left(1+q^{1+n}\right)} E_{q}\left(q^{\alpha}, q^{\beta}:: z\right)=\operatorname{S}_{0}^{\infty} e_{q}(-x) x^{\beta-1}{ }_{1} \Phi_{1}\left(q^{\alpha} ; 0 ;-x q^{-\beta} / z\right) d(q x) \text {. }
$$

This integral representation is interesting in the sense that it gives an alternative definition of the $E_{q}$-function corresponding to the alternative analogue $e_{q}(x)$ of the exponential function.

Next we deduce another definite integral involving in the integrand a basic analogue of the ${ }_{1} F_{1}$-function. In particular, let us consider the integral

$$
\frac{1}{1-q} \operatorname{S}_{0}^{1 / b} E_{q}(q b \lambda) E_{q}\left(\lambda a q^{\alpha-m-1}\right) \lambda^{\alpha+m-1}\left\{[1+\lambda]_{\alpha-m}\right\}^{-1} \Phi_{1}\left(-q^{1+m-\alpha} / \lambda, 0 ; q^{2 m+1} ; a b \lambda^{2} q^{\alpha-m-1}\right) d(q \lambda) \text {. }
$$

Expanding $E_{q}\left(\lambda a q^{\alpha-m-1}\right)$ and ${ }_{2} \Phi_{1}$, we have

$$
\begin{aligned}
& \frac{1}{1-q} \int_{0}^{1 / b} E_{q}(q b \lambda) \lambda^{\alpha+m-1}\left\{[1+\lambda]_{\alpha-m}\right\}^{-1} \\
& \quad \times \sum_{s=0}^{\infty} \sum_{t=0}^{\infty} \frac{(-)^{s} \lambda^{s} a^{s} q^{s(\alpha-m-1)+t s(s-1)}\left(-q^{1-\alpha+m} / \lambda\right)_{t}}{(q)_{s}(q)_{t}\left(q^{2 m+1}\right)_{t}} a^{t} b^{t} \lambda^{2 t} q^{t(\alpha-m-1)} d(q \lambda) .
\end{aligned}
$$

† For details see L. J. Slater, Proc. Cambridge Phil. Soc. 48 (1952), 578-82.

$\ddagger$ Slater, ibid. equation (13). 
Putting $t=r-s$, and changing the order of summation and integration, which is justified by absolute convergence of the series involved, for $R b>1,\left|a b q^{\alpha-m-1}\right|<1$, we have, after some simplification, that

$$
\frac{1}{1-q} \sum_{r=0}^{\infty} \sum_{s=0}^{r} \frac{(-)^{s} a^{r} b^{r-s} q^{s(\alpha-m+s-r-2)+t r(r-1)}}{(q)_{s}(q)_{r-s}\left(q^{2 m+1}\right)_{r-s}} \int_{0}^{1 / b} E_{q}(q b \lambda) \lambda^{\alpha+m+r-1} \Phi_{0}(\alpha-m+s-r ;-\lambda) d(q \lambda) \text {. }
$$

Changing the variable through the transformation $\lambda b=v$ and evaluating the integral by $[1,(3)]$, we get on simplification that the above is equal to

$$
\begin{aligned}
& \frac{b^{-\alpha-m}}{G(\alpha-m)} \sum_{r=0}^{\infty} \sum_{s=0}^{r} \frac{(-)^{r+s} b^{-s} a^{r}\left(q^{m-\alpha+1}\right)_{r}\left(q^{-r}\right)_{s}\left(q^{-2 m-r}\right)_{s}}{(q)_{s}\left(q^{\alpha-m-r}\right)_{s}(q)_{r}\left(q^{2 m+1}\right)_{r}} \\
& \times q^{(\alpha-m-1) r+s(\alpha+m+r)} E_{q}(\alpha-m+s-r, \alpha+m+r:: b) .
\end{aligned}
$$

Summing the $s$-series by $[1,(10)]$, we have finally that $(7)$ is equal to

$$
\frac{b^{-\alpha-m}}{G(\alpha-m)} E_{q}(\alpha-m, \alpha+m:: b)_{1} \Phi_{1}(\alpha+m ; 2 m+1 ; a),
$$

which gives the required result.

6. An $E_{q}$-function with negative argument. Consider the function

$$
\begin{aligned}
& E_{q}(1-\alpha, 1-\beta:: z / q)=\sum_{\alpha, \beta} \frac{G(1-\alpha) G(\alpha-\beta)}{G(1)} \prod \frac{\left(1+z^{-1} q^{2-\alpha+n}\right)\left(1+z q^{\alpha+n-1}\right)}{\left(1+z^{-1} q^{1+n}\right)\left(1+z q^{n}\right)} \\
& \times{ }_{1} \Phi_{1}\left(1-\alpha ; 1+\beta-\alpha ; z q^{\beta}\right) \text {. }
\end{aligned}
$$

Using the basic analogue of Kummer's formula, namely

$$
{ }_{1} \Phi_{1}\left(q^{a} ; q^{b} ; x\right)=\prod\left(1+x q^{a-b+n}\right)_{2} \Phi_{1}\left(q^{b-a}, 0 ; q^{b} ;-x q^{a-b}\right),
$$

we have, on simplification and transposition, that

$$
\begin{aligned}
& E_{q}\left(z^{-1} q\right) E_{q}(1-\alpha, 1-\beta::-z / q) \\
& \quad=\sum_{\alpha, \beta} \frac{G(1-\beta) G(\beta-\alpha)}{G(1)} \prod\left(1-z^{-1} q^{2-\beta+n}\right)\left(1-z q^{\beta+n-1}\right)_{2} \Phi_{1}(\alpha, 0 ; 1+\alpha-\beta ; z) .
\end{aligned}
$$

The formula (8) suggests the consideration of another $q$-analogue of the $E$-function in the form

$$
A_{2} \Phi_{1}(\alpha, 0 ; 1+\alpha-\beta ; z)+B_{2} \Phi_{1}(\beta, 0 ; 1+\beta-\alpha ; z),
$$

where $A$ and $B$ are suitable functions of $\alpha, \beta$ and $z$. This is natural to expect also, since, corresponding to the ${ }_{1} F_{1}$ function there can be two $q$-analogues, one with a quadratic power $q^{\operatorname{tn}(n-1)}$ in the argument and the other without it. Such a definition forms the subject matter of a subsequent communication.

It may also be interesting to note that the function $f(\alpha, \beta) \equiv E_{q}\left(z^{-1} q\right) E_{q}(1-\alpha, 1-\beta::-z / q)$ has properties very similar to the $E_{q}(\alpha, \beta:: z)$ function. For instance, it is easy to see that corresponding to (7) of [1] we have the recurrence relation

$$
\left(1-q^{1-\alpha}\right) f(\alpha, \beta)-f(\alpha-1, \beta)+\left(q^{2-\alpha} / z\right) f(\alpha-1, \beta-1)=0 .
$$




\section{REFERENCES}

1. R. P. Agarwal, A basic analogue of MacRobert's E-function, Proc. Glasgow Math. Assoc. 5 (1961), 4-7.

2. N. Agarwal, A $q$-analogue of MacRobert's generalized $E$-function, Ganita 12 (1961).

3. W. Hahn, Úber die höheren Heineschen Reihen und eine einheitliche Theorie der sogenannten speziellen Funktionen, Math. Nachr. 3 (1950), 257-294.

4. T. M. MacRobert, Induction proofs of the relation between certain asymptotic expansions and corresponding generalized hypergeometric series, Proc. Roy. Soc. Edinburgh 58 (1937), 1-13.

\section{LUCKNOW UNIVERSITY, LUCKNOW,}

INDIA 